Research Article

Anurak Thanyacharoen and Wutiphol Sintunavarat*

\title{
The new investigation of the stability of mixed type additive-quartic functional equations in non-Archimedean spaces
}

https://doi.org/10.1515/dema-2020-0009

received April 30, 2019; accepted April 17, 2020

Abstract: In this article, we prove the generalized Hyers-Ulam stability for the following additive-quartic functional equation:

$$
f(x+3 y)+f(x-3 y)+f(x+2 y)+f(x-2 y)+22 f(x)+24 f(y)=13[f(x+y)+f(x-y)]+12 f(2 y),
$$

where $f$ maps from an additive group to a complete non-Archimedean normed space.

Keywords: non-Archimedean normed spaces, linear functionals, functional equations

MSC 2020: 39B52, 39B55, 39B72, 47H10, 46H 25

\section{Introduction}

The study of stability problems for functional equations is one of the essential research in mathematics, which originated in issues related to applied mathematics. The first question concerning the stability of homomorphisms was given by Ulam [1] as follows.

Given a group $(G, *)$, a metric group $\left(G^{\prime}, \bullet\right)$ with the metric $d$, and a mapping $f$ from $G$ to $G^{\prime}$, does there exist $\delta>0$ such that

$$
d(f(x * y), f(x) \bullet f(y)) \leq \delta
$$

for all $x, y \in G$, then there exists a homomorphism $h: G \rightarrow G^{\prime}$ such that

$$
d(f(x), h(x)) \leq \epsilon
$$

for all $x \in G$ ?

The above question was solved in 1941 by Hyers [2] in the case of approximately additive mappings between two Banach spaces. The method used in the investigation of Hyers is called the direct method. In the same decade with the existing result of Hyers [2], Aoki [3] extended the Hyers' stability result for additive mappings by aiming an unbounded Cauchy difference. For almost 30 years later, the Hyers' stability result was improved from an assiduousness of Rassias [4]. The approximately additive condition of Hyers [2] was relaxed in his result as follows.

\footnotetext{
* Corresponding author: Wutiphol Sintunavarat, Department of Mathematics and Statistics, Faculty of Science and Technology, Thammasat University, Phatum Thani 12120, Thailand, e-mail: wutiphol@mathstat.sci.tu.ac.th

Anurak Thanyacharoen: Department of Mathematics and Statistics, Faculty of Science and Technology, Thammasat University, Phatum Thani 12120, Thailand, e-mail: ake_poiuy@yahoo.com
} 
Theorem 1.1. [4] Let $X$ and $Y$ be two Banach spaces. Suppose that $f: X \rightarrow Y$ is a function satisfying the following inequality:

$$
\|f(x+y)-f(x)-f(y)\| \leq \epsilon\left(\|x\|^{p}+\|y\|^{p}\right)
$$

for some $\epsilon \geq 0, p$ with $0 \leq p<1$ and for all $x, y \in X$. Then, there exists a unique additive function $A: X \rightarrow Y$ such that

$$
\|f(x)-A(x)\| \leq \frac{2 \epsilon}{2-2^{p}}\|x\|^{p}
$$

for each $x \in X$. If, in addition, $f(t x)$ is continuous at $t$ in a scalar field of $X$ for each fixed $x \in X$, then the function $A$ is linear.

The stability spectacle of Theorem 1.1 is called the generalized Hyers-Ulam stability. This glossary may also be used in other functional equations. Subsequently, a large number of investigations have been published in cohesion with several generalizations of stability results of Hyers [2], Aoki [3] and Rassias [4]. Moreover, many recent stability results had the inspiration from these fundamental stability results. For instance, Rassias [5] investigated the Hyers-Ulam stability for the quartic functional equation:

$$
f(x+2 y)+f(x-2 y)+6 f(x)=4[f(x+y)+f(x-y)+6 f(y)],
$$

where $f$ maps from a normed space into a Banach space. He also named the function satisfying (1.3) as a quartic function. The quartic functional equation (1.3) is one of the interesting topics in the stability theory for functional equations. It was employed by several researchers (see [6-10] and references therein).

Nowadays, the investigation of the stability in general content is essential and numerous. For instance, Chang et al. [11] investigated the stability results of the cosine-sine functional equation with involution in a commutative semigroup and a commutative group. Besides, Choi et al. [12] proved the stability results for functional equations arising from number theory and are connected with the characterizations of the determinant and permanent of two-by-two matrices. They considered a mapping from $\mathbb{R}^{2}$ into $\mathbb{R}$ by applying the important lemma of bounded mappings on a group.

Recently, Hengkrawit and Thanyacharoen [13] introduced the following additive-quartic functional equation:

$$
f(x+3 y)+f(x-3 y)+f(x+2 y)+f(x-2 y)+22 f(x)+24 f(y)=13[f(x+y)+f(x-y)]+12 f(2 y),
$$

where $f$ is a mapping from $\mathbb{R}$ to $\mathbb{R}$. The general solution of (1.4) on the oddness (or the evenness) of an unknown function is additive (or quartic). Based on this fact, the functional equation (1.4) is called a mixed type additive-quartic functional equation. Moreover, they also gave the Hyers-Ulam stability result of (1.4) on $\mathbb{R}$.

On the other hand, a special function on $\mathbb{Q}$ named a $p$-adic, or an ultrametric or simply a nonArchimedean absolute value on $\mathbb{Q}$ was first introduced by Hensel [14]. He also presented the idea of a nonArchimedean field which is a set $\mathbb{Q}$ together with the $p$-adic absolute value. Also, this concept can be extended to the general idea on the arbitrary field, and we can use this idea for defining the nonArchimedean vector space and the non-Archimedean norm. Based on all aforementioned ideas, many mathematicians investigated the stability results by applying such ideas. Here, we give some examples of these results.

- Arriola and Beyer [15] considered the stability of approximate additive mappings from the completion of a non-Archimedean field to $\mathbb{R}$.

- Kaiser [16] proved the stability problem Cauchy functional equation:

$$
f(x+y)=f(x)+f(y)
$$

on normed spaces over fields with valuation.

- Moslehian and Rassias [17] proved the generalized Hyers-Ulam stability of the Cauchy functional equation

$$
f(x+y)=f(x)+f(y)
$$


and the quadratic functional equation

$$
f(x+y)+f(x-y)=2 f(x)+2 f(y)
$$

in non-Archimedean normed spaces.

To the best of our knowledge, there is no discussion so far concerning the generalized Hyers-Ulam stability for the functional equation

$f(x+3 y)+f(x-3 y)+f(x+2 y)+f(x-2 y)+22 f(x)+24 f(y)=13[f(x+y)+f(x-y)]+12 f(2 y)$,

for all $x, y \in G$, where $f$ maps from an additive group $G$ to a complete non-Archimedean normed space. The main goal of this article is first to investigate the mentioned discussion by using two techniques containing the direct method and the fixed point method. Moreover, several particular cases of our main results are given.

\section{Preliminaries}

In this section, we will recall some basic concepts of non-Archimedean normed spaces.

Definition 2.1. [14] Let $\mathbb{K}$ be a field. A function $|\cdot|: \mathbb{K} \rightarrow[0, \infty)$ is called a non-Archimedean absolute value (non-Archimedean valuation) on $\mathbb{K}$ if the following conditions hold for all $a, b \in \mathbb{K}$ :

1. $|a|=0$ if and only if $a=0$;

2. $|a b|=|a||b|$;

3. $|a+b| \leq \max \{|a|,|b|\}$ (the strict triangle inequality).

Also, a field $\mathbb{K}$ with a non-Archimedean absolute value is called a non-Archimedean field.

In any non-Archimedean field $K$, we have $|1|=|-1|=1$ and $|n| \leq 1$ for each integer $n$. We always assume, in addition, that $|\cdot|$ is non-trivial, i.e., there exists an $a_{0} \in \mathbb{K}$ such that $\left|a_{0}\right| \neq 0,1$.

Definition 2.2. [14] Let $X$ be a vector space over a scalar field $\mathbb{K}$ with a non-Archimedean non-trivial valuation $\mid \cdot$. A function $\|\cdot\|: X \rightarrow \mathbb{R}$ is called a non-Archimedean norm (valuation) if it satisfies the following conditions:

1. $\|x\| \geq 0$ for all $x \in X$, and $\|x\|=0$ if and only if $x=0$;

2. $\|r x\|=|r|\|x\|$ for all $r \in \mathbb{K}$ and all $x \in X$;

3. the strong triangle inequality (ultrametric); namely, $\|x+y\| \leq \max \{\|x\|,\|y\|\}$ for all $x, y \in X$.

Also, $(X,\|\cdot\|)$ is called a non-Archimedean normed space.

Definition 2.3. [14] The sequence $\left\{x_{n}\right\}$ in a non-Archimedean normed space $(X,\|\cdot\|)$ is called convergent if for a given $\epsilon>0$ there are a positive integer $N$ and an $x \in X$ such that

$$
\left\|x_{n}-x\right\| \leq \epsilon
$$

for all $n \geq N$ and $x$ is called a limit of the sequence $\left\{x_{n}\right\}$, denoted by $\lim _{n \rightarrow \infty} x_{n}=x$.

Definition 2.4. [14] The sequence $\left\{x_{n}\right\}$ in a non-Archimedean normed space $(X,\|\cdot\|)$ is called Cauchy if for a given $\epsilon>0$ there is a positive integer $N$ such that

$$
\left\|x_{n}-x_{m}\right\| \leq \epsilon
$$

for all $n, m \geq N$.

In a non-Archimedean normed space $(X,\|\cdot\|)$, we have

$$
\left\|x_{n}-x_{m}\right\| \leq \max \left\{\left\|x_{j+1}-x_{j}\right\|: m \leq j \leq n-1\right\}
$$


for $n>m$. This yields the following proposition.

Proposition 2.5. [14] A sequence $\left\{x_{n}\right\}$ in a non-Archimedean space $(X,\|\cdot\|)$ is a Cauchy sequence if and only if the sequence $\left\{\left\|x_{n+1}-x_{n}\right\|\right\}$ converges to zero.

Next, we present the useful theorem which is the main tool to consider the stability result by using the fixed point method.

Theorem 2.6. [18] Let $(X, d)$ be a complete generalized metric space and let $J: X \rightarrow X$ be a strictly contractive mapping with some Lipschitz constant $L$ with $0<L<1$. Then, for each given element $x \in X$, either $d\left(J^{n} x, J^{n+1} x\right)=\infty$ for all nonnegative integers $n$ or there exists a positive integer $n_{0}$ such that

1. $d\left(J^{n} x, J^{n+1} x\right)<\infty$ for all $n \geq n_{0}$;

2. the sequence $\left\{J^{n} x\right\}$ converges to a fixed point $y^{*}$ of $J$;

3. $y^{*}$ is the unique fixed point of $J$ in the set $Y=\left\{y \in X \mid d\left(J^{n_{0}} x, y\right)<\infty\right\}$; and

4. $d\left(y, y^{*}\right) \leq \frac{1}{1-L} d(y, J y)$ for all $y \in Y$.

\section{Main results}

Throughout this section, let $G$ be an additive group, $\left(Y,\|\cdot\|_{Y}\right)$ be a non-Archimedean normed space and $f: G \rightarrow Y$ be a mapping. For each $x, y \in G$, we will use the following symbol:

$D f(x, y):=f(x+3 y)+f(x-3 y)+f(x+2 y)+f(x-2 y)+22 f(x)-13 f(x+y)-13 f(x-y)+24 f(y)-12 f(2 y)$.

First, we give two auxiliary lemmas for proving the first stability result via the direct method.

Lemma 3.1. Let $G$ be an additive group, $\left(Y,\|\cdot\|_{Y}\right)$ be a non-Archimedean normed space and $f: G \rightarrow Y$ be a mapping. If $f$ satisfies (1.5), then $f$ is of the form

$$
\begin{array}{r}
21 f(x+2 y)+21 f(x-2 y)-84 f(x+y)-84 f(x-y)+126 f(x) \\
+70 f(y)-30 f(-y)-33 f(2 y)+15 f(-2 y)-f(4 y)=0
\end{array}
$$

for all $x, y \in G$.

Proof. Suppose that $f$ satisfies (1.5). Substituting $x$ by $x+3 y$ into (1.5), we get

$f(x+6 y)+f(x)+f(x+5 y)+f(x+y)+22 f(x+3 y)-13 f(x+4 y)-13 f(x+2 y)+24 f(y)-12 f(2 y)=0$

for all $x, y \in X$. Substituting $x$ by $x+2 y$ into (1.5), we get

$f(x+5 y)+f(x-y)+f(x+4 y)+f(x)+22 f(x+2 y)-13 f(x+3 y)-13 f(x+y)+24 f(y)-12 f(2 y)=0$

for all $x, y \in X$. From (3.2) and (3.3), we obtain

$$
f(x+6 y)-14 f(x+4 y)+35 f(x+3 y)-35 f(x+2 y)+14 f(x+y)-f(x-y)=0
$$

for all $x, y \in X$. Substituting $y$ by $-y$ into (3.4), we get

$$
f(x-6 y)-14 f(x-4 y)+35 f(x-3 y)-35 f(x-2 y)+14 f(x-y)-f(x+y)=0
$$

for all $x, y \in X$. From (3.4) and (3.5), we obtain

$$
\begin{array}{r}
f(x+6 y)+f(x-6 y)-14 f(x+4 y)-14 f(x-4 y)+35 f(x+3 y)+35 f(x-3 y) \\
-35 f(x+2 y)-35 f(x-2 y)+13 f(x+y)+13 f(x-y)=0
\end{array}
$$

for all $x, y \in X$. Substituting $y$ by $2 y$ into (1.5), we have 


$$
\begin{array}{r}
f(x+6 y)+f(x-6 y)+f(x+4 y)+f(x-4 y)+22 f(x)-13 f(x+2 y) \\
-13 f(x-2 y)+24 f(2 y)-12 f(4 y)=0
\end{array}
$$

for all $x, y \in X$. From (3.6) and (3.7), we obtain

$$
\begin{array}{r}
15 f(x+4 y)+15 f(x-4 y)-35 f(x+3 y)-35 f(x-3 y)+22 f(x+2 y)+22 f(x-2 y) \\
-13 f(x+y)-13 f(x-y)+22 f(x)+24 f(2 y)-12 f(4 y)=0
\end{array}
$$

for all $x, y \in X$. Substituting $x$ by $x+y$ into (1.5), we get

$$
\begin{array}{r}
f(x+4 y)+f(x-2 y)+f(x+3 y)+f(x-y)+22 f(x+y)-13 f(x+2 y) \\
-13 f(x)+24 f(y)-12 f(2 y)=0
\end{array}
$$

for all $x, y \in X$. Substituting $y$ by $-y$ into (3.9), we have

$$
\begin{array}{r}
f(x-4 y)+f(x+2 y)+f(x-3 y)+f(x+y)+22 f(x-y)-13 f(x-2 y) \\
-13 f(x)+24 f(-y)-12 f(-2 y)=0
\end{array}
$$

for all $x, y \in X$. From (3.9) and (3.10), we obtain

$$
\begin{array}{r}
f(x+4 y)+f(x-4 y)+f(x+3 y)+f(x-3 y)-12 f(x+2 y)-12 f(x-2 y)+23 f(x+y) \\
+23 f(x-y)-26 f(x)+24 f(y)-12 f(2 y)+24 f(-y)-12 f(-2 y)=0,
\end{array}
$$

thus

$$
\begin{array}{r}
15 f(x+4 y)+15 f(x-4 y)+15 f(x+3 y)+15 f(x-3 y)-180 f(x+2 y)-180 f(x-2 y)+345 f(x+y) \\
+345 f(x-y)-390 f(x)+360 f(y)+360 f(-y)-180 f(2 y)-180 f(-2 y)=0
\end{array}
$$

for all $x, y \in X$. From (3.8) and (3.12), we obtain

$$
\begin{array}{r}
50 f(x+3 y)+50 f(x-3 y)-202 f(x+2 y)-202 f(x-2 y)+358 f(x+y)+358 f(x-y)-412 f(x) \\
+360 f(y)+360 f(-y)-204 f(2 y)-180 f(-2 y)+12 f(4 y)=0
\end{array}
$$

for all $x, y \in X$. From (1.5), we get

$$
\begin{array}{r}
50 f(x+3 y)+50 f(x-3 y)+50 f(x+2 y)+50 f(x-2 y)-650 f(x+y) \\
-650 f(x-y)+1100 f(x)+1200 f(y)-600 f(2 y)=0
\end{array}
$$

for all $x, y \in X$. From (3.13) and (3.14), we have

$$
\begin{array}{r}
252 f(x+2 y)+252 f(x-2 y)-1008 f(x+y)-1008 f(x-y)+1512 f(x) \\
+840 f(y)-360 f(-y)-396 f(2 y)+180 f(-2 y)-12 f(4 y)=0,
\end{array}
$$

thus

$$
\begin{array}{r}
21 f(x+2 y)+21 f(x-2 y)-84 f(x+y)-84 f(x-y)+126 f(x) \\
+70 f(y)-30 f(-y)-33 f(2 y)+15 f(-2 y)-f(4 y)=0
\end{array}
$$

for all $x, y \in X$.

Lemma 3.2. Let $G$ be an additive group, $\left(Y,\|\cdot\|_{Y}\right)$ be a non-Archimedean normed space and $f: G \rightarrow Y$ be a mapping satisfying (1.5). Then, the following assertions hold:

(1) $f$ is even if and only if $f$ is quartic;

(2) $f$ is odd if and only if $f$ is additive.

Proof. Replacing $x$ and $y$ by 0 in (1.5), we have $f(0)=0$.

(1) For the first implication, we suppose that $f$ is even. By Lemma 3.1, $f$ is of the form (3.1). Putting $x=0$ in (3.1), since $f$ is even and $f(0)=0$, we obtain 


$$
24 f(2 y)-128 f(y)-f(4 y)=0
$$

for all $y \in X$. It follows the proof of Lemma 3.1 that

$$
\begin{array}{r}
15 f(x+4 y)+15 f(x-4 y)-35 f(x+3 y)-35 f(x-3 y)+22 f(x+2 y)+22 f(x-2 y) \\
-13 f(x+y)-13 f(x-y)+22 f(x)+24 f(2 y)-12 f(4 y)=0
\end{array}
$$

for all $x, y \in X$. Putting $x=0$ in (3.18), since $f$ is even and $f(0)=0$, we obtain

$$
30 f(4 y)-70 f(3 y)+44 f(2 y)-26 f(y)+24 f(2 y)-12 f(4 y)=0,
$$

thus

$$
9 f(4 y)-35 f(3 y)+34 f(2 y)-13 f(y)=0
$$

for all $y \in X$. From (3.17), we get

$$
216 f(2 y)-1152 f(y)-9 f(4 y)=0
$$

for all $y \in X$. From (3.19) and (3.20), we have

$$
-35 f(3 y)+250 f(2 y)-1165 f(y)=0
$$

for all $y \in X$. Letting $x=0$ in (1.5), since $f$ is even and $f(0)=0$, we obtain

$$
f(3 y)-5 f(2 y)-f(y)=0,
$$

thus

$$
35 f(3 y)-175 f(2 y)-35 f(y)=0
$$

for all $y \in X$. From (3.21) and (3.22), we get

$$
f(2 y)=16 f(y)
$$

for all $y \in X$. From (3.1), we have

$$
f(x+2 y)+f(x-2 y)-4 f(x+y)-4 f(x-y)+6 f(x)-24 f(y)=0
$$

for all $x, y \in X$, so $f$ is a quartic mapping. Finally, it is easy to prove that the second implication holds. This completes the proof.

(2) For the first implication, we suppose that $f$ is odd. By Lemma 3.1, $f$ is of the form (3.1). Substituting $y$ by $-y$ into (1.5), since $f$ is odd, we get

$$
\begin{array}{r}
f(x+3 y)+f(x-3 y)+f(x+2 y)+f(x-2 y)+22 f(x)-13 f(x+y)-13 f(x-y) \\
-24 f(y)+12 f(2 y)=0
\end{array}
$$

for all $x, y \in X$. From (1.5) and (3.25), we obtain

$$
f(2 y)=2 f(y)
$$

for all $y \in X$. From (3.1) and (3.26), we have

$$
f(x+2 y)+f(x-2 y)-4 f(x+y)-4 f(x-y)+6 f(x)=0
$$

for all $x, y \in X$. Substituting $x$ by $2 x$ into (3.27) and using (3.26), we get

$$
2 f(2 x+y)+2 f(2 x-y)-f(x+y)-f(x-y)-6 f(x)=0
$$

for all $x, y \in X$. Interchanging $x$ into $y$ in (3.27), we have

$$
f(2 x+y)-f(2 x-y)-4 f(x+y)+4 f(x-y)+6 f(y)=0
$$

for all $x, y \in X$. Substituting $y$ by $-y$ into (3.29), we get

$$
f(2 x-y)-f(2 x+y)-4 f(x-y)+4 f(x+y)-6 f(y)=0
$$


for all $x, y \in X$. Replacing $x$ by $2 x$ in (3.28) and using (3.31), we get

$$
2 f(4 x+y)+2 f(4 x-y)-f(2 x+y)-f(2 x-y)-12 f(x)=0
$$

for all $x, y \in X$. From (3.28) and (3.31), we obtain

$$
4 f(4 x+y)+4 f(4 x-y)-f(x+y)-f(x-y)-30 f(x)=0
$$

for all $x, y \in X$. Substituting $y$ by $y+2 x$ into (3.28), we have

$$
4 f(4 x+y)-4 f(y)-2 f(3 x+y)+2 f(x+y)-12 f(x)=0
$$

for all $x, y \in X$. Replacing $y$ by $-y$ in (3.33), we get

$$
4 f(4 x-y)+4 f(y)-2 f(3 x-y)+2 f(x-y)-12 f(x)=0
$$

for all $x, y \in X$. From (3.33) and (3.34), we obtain

$$
4 f(4 x+y)+4 f(4 x-y)-2 f(3 x+y)-2 f(3 x-y)+2 f(x+y)+2 f(x-y)-24 f(x)=0
$$

for all $x, y \in X$. From (3.32) and (3.35), we obtain

$$
2 f(3 x+y)+2 f(3 x-y)-3 f(x+y)-3 f(x-y)-6 f(x)=0
$$

for all $x, y \in X$. Substituting $y$ by $x-y$ into (3.28), we have

$$
2 f(3 x-y)+2 f(x+y)-f(2 x-y)-f(y)-6 f(x)=0
$$

for all $x, y \in X$. Substituting $y$ by $x+y$ into (3.28), we get

$$
2 f(3 x+y)+2 f(x-y)-f(2 x+y)+f(y)-6 f(x)=0
$$

for all $x, y \in X$. From (3.37) and (3.38), we obtain

$$
2 f(3 x+y)+2 f(3 x-y)-f(2 x+y)-f(2 x-y)+2 f(x+y)+2 f(x-y)-12 f(x)=0
$$

for all $x, y \in X$. From (3.28) and (3.39), we have

$$
4 f(3 x+y)+4 f(3 x-y)+3 f(x+y)+3 f(x-y)-30 f(x)=0
$$

for all $x, y \in X$. From (3.36) and (3.40), we have

$$
f(x+y)+f(x-y)-2 f(x)=0
$$

for all $x, y \in X$. Interchanging $x$ into $y$ in (3.41), we get

$$
f(x+y)-f(x-y)-2 f(y)=0
$$

for all $x, y \in X$. From (3.41) and (3.42), we obtain

$$
f(x+y)=f(x)+f(y)
$$

for all $x, y \in X$, so $f$ is an additive mapping. Finally, it is easy to prove that the second implication holds. This completes the proof.

Next, we are going to consider the stability of the additive-quartic functional equation (1.5).

Theorem 3.3. Let $G$ be an additive group, $\left(Y,\|\cdot\|_{Y}\right)$ be a complete non-Archimedean normed space and $\phi: G^{2} \rightarrow[0, \infty)$ be a function such that

$$
\lim _{n \rightarrow \infty} \frac{\phi\left(2^{n} x, 2^{n} y\right)}{2^{n}}=0
$$

for all $x, y \in G$, and the following limits exist for each $x \in G$ : 


$$
\lim _{n \rightarrow \infty} \max \left\{\frac{\psi\left(0,2^{j} x\right)}{2^{j}}: 0 \leq j<n\right\}
$$

and

$$
\lim _{n \rightarrow \infty} \max \left\{\frac{\tilde{\psi}\left(2^{j} x\right)}{2^{j}}: 0 \leq j<n\right\}
$$

where $\psi(x, y):=\max \{\phi(x, y), \phi(-x,-y)\}$ for all $x, y \in G$ and

$$
\tilde{\psi}(x):=\max \left\{\psi(3 x, x), \psi(2 x, x), \frac{\psi(0,2 x)}{2}, 9 \psi(x, x), 22 \psi(0, x)\right\}
$$

for all $x \in G$. Suppose that $f: G \rightarrow Y$ is a mapping satisfying $f(0)=0$ and

$$
\|D f(x, y)\|_{Y} \leq \phi(x, y)
$$

for all $x, y \in G$. Then, there exist a unique quartic function $Q: G \rightarrow Y$ and a unique additive function $A: G \rightarrow Y$ such that

$$
\begin{aligned}
& \left\|\frac{f(x)+f(-x)}{2}-Q(x)\right\|_{Y} \leq \frac{1}{960} \lim _{n \rightarrow \infty} \max \left\{\frac{\tilde{\psi}\left(2^{j} x\right)}{16^{j}}: 0 \leq j<n\right\}, \\
& \left\|\frac{f(x)-f(-x)}{2}-A(x)\right\|_{Y} \leq \frac{1}{48} \lim _{n \rightarrow \infty} \max \left\{\frac{\psi\left(0,2^{j} x\right)}{2^{j}}: 0 \leq j<n\right\}
\end{aligned}
$$

and

$$
\|f(x)-Q(x)-A(x)\|_{Y} \leq \max \left\{\frac{1}{960} \lim _{n \rightarrow \infty} \max \left\{\frac{\tilde{\psi}\left(2^{j} x\right)}{16^{j}}: 0 \leq j<n\right\}, \frac{1}{48} \lim _{n \rightarrow \infty} \max \left\{\frac{\psi\left(0,2^{j} x\right)}{2^{j}}: 0 \leq j<n\right\}\right\}(3.5
$$

for all $x \in G$. Moreover, if

$$
\lim _{i \rightarrow \infty} \lim _{n \rightarrow \infty} \max \left\{\frac{\tilde{\psi}\left(2^{j} x\right)}{16^{j}}: 0 \leq i \leq j<n+i\right\}=0
$$

and

$$
\lim _{i \rightarrow \infty} \lim _{n \rightarrow \infty} \max \left\{\frac{\psi\left(0,2^{j} x\right)}{2^{j}}: 0 \leq i \leq j<n+i\right\}=0
$$

for all $x \in G$, then $Q$ and $A$ are the unique quartic and additive functions satisfying (3.49) and (3.50), respectively.

Proof. Let $f_{1}: G \rightarrow Y$ be the function defined by $f_{1}(x):=\frac{f(x)+f(-x)}{2}$ for all $x \in G$. Then, $f_{1}(0)=0$ and $f_{1}(x)=f_{1}(-x)$ for all $x \in G$. Replacing $x$ by $-x$ and $y$ by $-y$ into (3.48), we have

$$
\|D f(-x,-y)\|_{Y} \leq \phi(-x,-y)
$$

for all $x, y \in G$. From (3.48) and (3.54), we obtain

$$
\left\|D f_{1}(x, y)\right\|_{Y} \leq \frac{1}{2} \max \{\phi(x, y), \phi(-x,-y)\}=\frac{\psi(x, y)}{2}
$$

for all $x, y \in G$. Letting $x=3 y$ in (3.55) and using $f_{1}(0)=0$, we get

$$
\left\|f_{1}(6 y)+f_{1}(5 y)-13 f_{1}(4 y)+22 f_{1}(3 y)-25 f_{1}(2 y)+25 f_{1}(y)\right\|_{Y} \leq \frac{\psi(3 y, y)}{2}
$$

for all $y \in G$. Letting $x=2 y$ in (3.55) and using $f_{1}(0)=0$, we have 


$$
\left\|f_{1}(5 y)+f_{1}(4 y)-13 f_{1}(3 y)+10 f_{1}(2 y)+12 f_{1}(y)\right\|_{Y} \leq \frac{\psi(2 y, y)}{2}
$$

for all $y \in G$. From (3.56) and (3.57), we obtain

$$
\left\|f_{1}(6 y)-14 f_{1}(4 y)+35 f_{1}(3 y)-35 f_{1}(2 y)+13 f_{1}(y)\right\|_{Y} \leq \frac{1}{2} \max \{\psi(3 y, y), \psi(2 y, y)\}
$$

for all $y \in G$. Letting $x=0$ in (3.55), we get

$$
\left\|2 f_{1}(3 y)-10 f_{1}(2 y)-2 f_{1}(y)\right\|_{Y} \leq \frac{\psi(0, y)}{2},
$$

that is,

$$
\left\|f_{1}(3 y)-5 f_{1}(2 y)-f_{1}(y)\right\|_{Y} \leq \frac{1}{4} \psi(0, y)
$$

for all $y \in G$. Replacing $y$ by $2 y$ in (3.60), we obtain

$$
\left\|f_{1}(6 y)-5 f_{1}(4 y)-f_{1}(2 y)\right\|_{Y} \leq \frac{\psi(0,2 y)}{4}
$$

for all $y \in G$. From (3.58) and (3.61), we obtain

$$
\left\|-9 f_{1}(4 y)+35 f_{1}(3 y)-34 f_{1}(2 y)+13 f_{1}(y)\right\|_{Y} \leq \frac{1}{2} \max \left\{\psi(3 y, y), \psi(2 y, y), \frac{\psi(0,2 y)}{2}\right\}
$$

for all $y \in G$. Letting $x=y$ in (3.55), we get

$$
\left\|f_{1}(4 y)+f_{1}(3 y)-24 f_{1}(2 y)+47 f_{1}(y)\right\|_{Y} \leq \frac{\psi(y, y)}{2},
$$

thus

$$
\left\|9 f_{1}(4 y)+9 f_{1}(3 y)-216 f_{1}(2 y)+423 f_{1}(y)\right\|_{Y} \leq \frac{9 \psi(y, y)}{2}
$$

for all $y \in G$. From (3.62) and (3.64), we obtain

$$
\left\|44 f_{1}(3 y)-250 f_{1}(2 y)+436 f_{1}(y)\right\|_{Y} \leq \frac{1}{2} \max \left\{\psi(3 y, y), \psi(2 y, y), \frac{\psi(0,2 y)}{2}, 9 \psi(y, y)\right\}
$$

for all $y \in G$. From (3.60), we get

$$
\left\|44 f_{1}(3 y)-220 f_{1}(2 y)-44 f_{1}(y)\right\|_{Y} \leq 11 \psi(0, y)
$$

for all $y \in G$. From (3.65) and (3.66), we obtain

$$
\left\|-30 f_{1}(2 y)+480 f_{1}(y)\right\|_{Y} \leq \frac{1}{2} \max \left\{\psi(3 y, y), \psi(2 y, y), \frac{\psi(0,2 y)}{2}, 9 \psi(y, y), 22 \psi(0, y)\right\}
$$

for all $y \in G$. Thus, we have

$$
\left\|f_{1}(y)-\frac{f_{1}(2 y)}{16}\right\|_{Y} \leq \frac{1}{960} \max \left\{\psi(3 y, y), \psi(2 y, y), \frac{\psi(0,2 y)}{2}, 9 \psi(y, y), 22 \psi(0, y)\right\}=\frac{1}{960} \tilde{\psi}(y)
$$

for all $y \in G$. Replacing $y$ by $2^{n-1} y$ in (3.68) and dividing by $16^{n-1}$, we get

$$
\left\|\frac{f_{1}\left(2^{n-1} y\right)}{16^{n-1}}-\frac{f_{1}\left(2^{n} y\right)}{16^{n}}\right\|_{Y} \leq \frac{1}{960} \frac{\tilde{\psi}\left(2^{n-1} y\right)}{16^{n-1}}
$$

for all $y \in G$. It follows from (3.68) and (3.69) that the sequence $\left\{\frac{f_{1}\left(2^{n} y\right)}{16^{n}}\right\}$ is a Cauchy sequence in $Y$. Since $Y$ is complete, there exists a function $Q: G \rightarrow Y$ such that 


$$
Q(y):=\lim _{n \rightarrow \infty} \frac{f_{1}\left(2^{n} y\right)}{16^{n}}
$$

for all $y \in G$. From (3.69) and the strong triangle inequality, we obtain

$$
\left\|f_{1}(y)-\frac{f_{1}\left(2^{n} y\right)}{16^{n}}\right\|_{Y} \leq \frac{1}{960} \max \left\{\frac{\tilde{\psi}\left(2^{j} y\right)}{16^{j}}: 0 \leq j<n\right\}
$$

for all $n \in \mathbb{N}$ and all $y \in G$. Taking $n \rightarrow \infty$ in the above inequality, it yields that

$$
\left\|f_{1}(y)-Q(y)\right\|_{Y} \leq \frac{1}{960} \lim _{n \rightarrow \infty} \max \left\{\frac{\tilde{\psi}\left(2^{j} y\right)}{16^{j}}: 0 \leq j<n\right\}
$$

for all $y \in G$. By (3.44) and (3.48), we get

$$
\begin{aligned}
\left\|\frac{D f_{1}\left(2^{n} x, 2^{n} y\right)}{16^{n}}\right\|_{Y} & =\frac{1}{2}\left\|\frac{D f\left(2^{n} x, 2^{n} y\right)+D f\left(-2^{n} x,-2^{n} y\right)}{16^{n}}\right\|_{Y} \\
& \leq \frac{1}{2} \max \left\{\frac{\left\|D f\left(2^{n} x, 2^{n} y\right)\right\|_{Y}}{16^{n}}, \frac{\left\|D f\left(-2^{n} x,-2^{n} y\right)\right\|_{Y}}{16^{n}}\right\} \\
& \leq \frac{1}{2} \max \left\{\frac{\phi\left(2^{n} x, 2^{n} y\right)}{16^{n}}, \frac{\phi\left(-2^{n} x,-2^{n} y\right)}{16^{n}}\right\}
\end{aligned}
$$

for all $x, y \in G$. From the last inequality, letting $n \rightarrow \infty$, we obtain

$$
D Q(x, y)=0
$$

for all $x, y \in G$. Since $f_{1}(0)=0$, we obtain $Q(0)=0$. Since $f_{1}$ is even,

$$
\|Q(x)-Q(-x)\|_{Y}=\lim _{n \rightarrow \infty}\left\|Q(x)-\frac{f_{1}\left(-2^{n} x\right)}{16^{n}}\right\|_{Y}=\lim _{n \rightarrow \infty}\left\|Q(x)-\frac{f_{1}\left(2^{n} x\right)}{16^{n}}\right\|_{Y}=0
$$

for all $x \in G$ and so $Q$ is even. By Lemma 3.2, we get $Q$ is a quartic mapping.

Let $f_{2}: G \rightarrow Y$ be the function defined by $f_{2}(x):=\frac{f(x)-f(-x)}{2}$ for all $x \in G$. Then, $f_{2}(0)=0$ and $f_{2}(-x)=-f_{2}(x)$ for all $x \in G$. From (3.48), we obtain

$$
\left\|D f_{2}(x, y)\right\|_{Y} \leq \frac{\psi(x, y)}{2}
$$

for all $x, y \in G$. Letting $x=0$ in (3.74), and using $f_{2}$ is odd and $f_{2}(0)=0$, we have

$$
\left\|24 f_{2}(y)-12 f_{2}(2 y)\right\|_{Y} \leq \frac{\psi(0, y)}{2},
$$

that is,

$$
\left\|f_{2}(y)-\frac{f_{2}(2 y)}{2}\right\|_{Y} \leq \frac{\psi(0, y)}{48}
$$

for all $y \in G$. Replacing $y$ by $2^{n-1} y$ in (3.76) and dividing by $2^{n-1}$, we get

$$
\left\|\frac{f_{2}\left(2^{n-1} y\right)}{2^{n-1}}-\frac{f_{2}\left(2^{n} y\right)}{2^{n}}\right\|_{Y} \leq \frac{1}{48} \frac{\psi\left(0,2^{n-1} y\right)}{2^{n-1}}
$$

for all $y \in G$. It follows from (3.76) and (3.77) that the sequence $\left\{\frac{f_{2}\left(2^{n} y\right)}{2^{n}}\right\}$ is a Cauchy sequence in $Y$. Since $Y$ is complete, there exists a function $A: G \rightarrow Y$ such that

$$
A(y):=\lim _{n \rightarrow \infty} \frac{f_{2}\left(2^{n} y\right)}{2^{n}}
$$

for all $y \in G$. From (3.77) and the strong triangle inequality, we have 


$$
\left\|f_{2}(2 y)-\frac{f_{2}\left(2^{n} y\right)}{2^{n}}\right\|_{Y} \leq \frac{1}{48} \max \left\{\frac{\psi\left(0,2^{j} y\right)}{2^{j}}: 0 \leq j<n\right\}
$$

for all $n \in \mathbb{N}$ and all $y \in G$. Taking $n \rightarrow \infty$ in the above inequality, it yields that

$$
\left\|f_{2}(2 y)-A(y)\right\|_{Y} \leq \frac{1}{48} \lim _{n \rightarrow \infty} \max \left\{\frac{\psi\left(0,2^{j} y\right)}{2^{j}}: 0 \leq j<n\right\}
$$

for all $y \in G$. By (3.44) and (3.48), we have

$$
\begin{aligned}
\left\|\frac{D f_{2}\left(2^{n} x, 2^{n} y\right)}{2^{n}}\right\|_{Y} & =\frac{1}{2}\left\|\frac{D f\left(2^{n} x, 2^{n} y\right)+D f\left(-2^{n} x,-2^{n} y\right)}{2^{n}}\right\|_{Y} \\
& \leq \frac{1}{2} \max \left\{\frac{\left\|D f\left(2^{n} x, 2^{n} y\right)\right\|_{Y}}{2^{n}}, \frac{\left\|D f\left(-2^{n} x,-2^{n} y\right)\right\|_{Y}}{2^{n}}\right\} \\
& \leq \frac{1}{2} \max \left\{\frac{\phi\left(2^{n} x, 2^{n} y\right)}{2^{n}}, \frac{\phi\left(-2^{n} x,-2^{n} y\right)}{2^{n}}\right\}
\end{aligned}
$$

for all $x, y \in G$. From the last inequality, letting $n \rightarrow \infty$, we get

$$
D A(x, y)=0
$$

for all $x, y \in G$. Since $f_{2}(0)=0$, we obtain $A(0)=0$. Since $f_{2}$ is odd,

$$
\|A(x)+A(-x)\|_{Y}=\lim _{n \rightarrow \infty}\left\|A(x)+\frac{f_{2}\left(-2^{n} x\right)}{2^{n}}\right\|_{Y}=\lim _{n \rightarrow \infty}\left\|A(x)-\frac{f_{2}\left(2^{n} x\right)}{2^{n}}\right\|_{Y}=0
$$

for all $x \in G$ and so $A$ is odd. By Lemma 3.2, we have $A$ is an additive mapping. Since $f(x)=f_{1}(x)+f_{2}(x)$ for all $x \in G$, from (3.72) and (3.80) it follows that

$$
\begin{aligned}
\|f(x)-Q(x)-A(x)\|_{Y} & =\left\|f_{1}(x)+f_{2}(x)-Q(x)-A(x)\right\|_{Y} \\
& \leq \max \left\{\left\|f_{1}(x)-Q(x)\right\|_{Y},\left\|f_{2}(x)-A(x)\right\|_{Y}\right\} \\
& \leq \max \left\{\frac{1}{960} \lim _{n \rightarrow \infty} \max \left\{\frac{\tilde{\psi}\left(2^{j} x\right)}{16^{j}}: 0 \leq j<n\right\}, \frac{1}{48} \lim _{n \rightarrow \infty} \max \left\{\frac{\psi\left(0,2^{j} x\right)}{2^{j}}: 0 \leq j<n\right\}\right\}
\end{aligned}
$$

for all $x \in G$.

Next, we will claim the uniqueness of $Q$ and $A$. Suppose that there exist a quartic mapping $Q^{\prime}: G \rightarrow Y$ satisfying (3.49) and an additive mapping $A^{\prime}: G \rightarrow Y$ (3.50). By using (3.49) and (3.52), we have

$$
\begin{aligned}
\left\|Q(x)-Q^{\prime}(x)\right\|_{Y} & =\lim _{i \rightarrow \infty}\left\|\frac{Q\left(2^{i} x\right)}{16^{i}}-\frac{Q^{\prime}\left(2^{i} x\right)}{16^{i}}\right\|_{Y} \\
& =\lim _{i \rightarrow \infty} \frac{1}{16^{i}}\left\|Q\left(2^{i} x\right)-f_{1}\left(2^{i} x\right)-Q^{\prime}\left(2^{i} x\right)+f_{1}\left(2^{i} x\right)\right\|_{Y} \\
& \leq \lim _{i \rightarrow \infty} \frac{1}{16^{i}} \max \left\{\left\|Q\left(2^{i} x\right)-f_{1}\left(2^{i} x\right)\right\|_{Y},\left\|Q^{\prime}\left(2^{i} x\right)-f_{1}\left(2^{i} x\right)\right\|_{Y}\right\} \\
& \leq \frac{1}{960} \lim _{i \rightarrow \infty} \lim _{n \rightarrow \infty} \max \left\{\frac{\tilde{\psi}\left(2^{j} x\right)}{16^{j}}: 0 \leq i \leq j<n+i\right\}
\end{aligned}
$$

for all $x \in G$. Since the right-hand side of the above inequality converges to 0 , we obtain $Q(x)=Q^{\prime}(x)$ for all $x \in G$, that is $Q=Q^{\prime}$. Similarly, we get $A=A^{\prime}$.

Corollary 3.4. Let $G$ be a normed space, $\left(Y,\|\cdot\|_{Y}\right)$ be a complete non-Archimedean normed space. Suppose that $f: G \rightarrow Y$ is a mapping satisfying $f(0)=0$ and there are a positive real number $\lambda$ and a real number $s<1$ such that

$$
\|D f(x, y)\|_{Y} \leq \lambda\left(\|x\|^{s}+\|y\|^{s}\right)
$$


for all $x, y \in G-\{0\}$. Then, there exist a unique quartic mapping $Q: G \rightarrow Y$ and a unique additive mapping $A: G \rightarrow Y$ such that

$$
\begin{aligned}
& \left\|\frac{f(x)+f(-x)}{2}-Q(x)\right\|_{Y} \\
& \quad \leq \frac{\lambda}{960} \lim _{n \rightarrow \infty} \max \left\{\frac{\left(3^{s}+1\right) 2^{j s}\|x\|^{s}}{16^{j}}, \frac{\left(2^{s}+1\right) 2^{j s}\|x\|^{s}}{16^{j}}, \frac{2^{s} 2^{j s}\|x\|^{s}}{16^{j}}, \frac{2^{j s} 18\|x\|^{s}}{16^{j}}, \frac{2^{j s} 22\|x\|^{s}}{16^{j}}: 0 \leq j<n\right\}, \\
& \left\|\frac{f(x)-f(-x)}{2}-A(x)\right\|_{Y} \leq \frac{\lambda}{48} \lim _{n \rightarrow \infty} \max \left\{\frac{2^{j s}\|x\|^{s}}{2^{j}}: 0 \leq j<n\right\}
\end{aligned}
$$

and

$$
\begin{aligned}
\|f(x)-Q(x)-A(x)\|_{Y} \leq & \max \left\{\frac { \lambda } { 9 6 0 } \operatorname { l i m } _ { n \rightarrow \infty } \operatorname { m a x } \left\{\frac{\left(3^{s}+1\right) 2^{j s}\|x\|^{s}}{16^{j}}, \frac{\left(2^{s}+1\right) 2^{j s}\|x\|^{s}}{16^{j}}, \frac{2^{s} 2^{j s}\|x\|^{s}}{16^{j}},\right.\right. \\
& \left.\left.\frac{2^{j s} 18\|x\|^{s}}{16^{j}}, \frac{2^{j s} 22\|x\|^{s}}{16^{j}}: 0 \leq j<n\right\}, \frac{\lambda}{48} \lim _{n \rightarrow \infty} \max \left\{\frac{2^{j s}\|x\|^{s}}{2^{j}}: 0 \leq j<n\right\}\right\}
\end{aligned}
$$

for all $x \in G-\{0\}$.

Proof. Define a mapping $\phi: G^{2} \rightarrow[0, \infty)$ by

$$
\phi(x, y)= \begin{cases}0, & \text { if } x=0 \text { or } y=0 \\ \lambda\left(\|x\|^{s}+\|y\|^{s}\right), & \text { other }\end{cases}
$$

for all $x, y \in G$.

From the definition of $\phi$, for each $x \in G$, we obtain

$$
\lim _{n \rightarrow \infty} \max \left\{\frac{\psi\left(0,2^{j} x\right)}{2^{j}}: 0 \leq j<n\right\}
$$

and

$$
\lim _{n \rightarrow \infty} \max \left\{\frac{\tilde{\psi}\left(2^{j} x\right)}{2^{j}}: 0 \leq j<n\right\}
$$

exist.

For $x=0$ or $y=0$, we get

$$
\lim _{n \rightarrow \infty} \frac{\phi\left(2^{n} x, 2^{n} y\right)}{2^{n}}=0
$$

For each $x, y \in G-\{0\}$, we have

$$
\lim _{n \rightarrow \infty} \frac{\phi\left(2^{n} x, 2^{n} y\right)}{2^{n}}=\lim _{n \rightarrow \infty} \frac{\lambda\left(\left\|2^{n} x\right\|^{s}+\left\|2^{n} y\right\|^{s}\right)}{2^{n}}=\lambda \lim _{n \rightarrow \infty} \frac{2^{n s}\left(\|x\|^{s}+\|y\|^{s}\right)}{2^{n}}=\lambda\left(\|x\|^{s}+\|y\|^{s}\right) \lim _{n \rightarrow \infty}\left(2^{s-1}\right)^{n}=0 .
$$

From (3.85) and (3.86), we have

$$
\lim _{n \rightarrow \infty} \frac{\phi\left(2^{n} x, 2^{n} y\right)}{2^{n}}=0
$$

for all $x, y \in G$.

For $x=0$, we have

$$
\lim _{i \rightarrow \infty} \lim _{n \rightarrow \infty} \max \left\{\frac{\tilde{\psi}\left(2^{j} x\right)}{16^{j}}: 0 \leq i \leq j<n+i\right\}=0
$$


and

$$
\lim _{i \rightarrow \infty} \lim _{n \rightarrow \infty} \max \left\{\frac{\psi\left(0,2^{j} x\right)}{2^{j}}: 0 \leq i \leq j<n+i\right\}=0 .
$$

For $x \neq 0$, we have

$$
\begin{aligned}
& \lim _{i \rightarrow \infty} \lim _{n \rightarrow \infty} \max \left\{\frac{\tilde{\psi}\left(2^{j} x\right)}{16^{j}}: 0 \leq i \leq j<n+i\right\} \\
& =\frac{\lambda}{960} \lim _{i \rightarrow \infty} \lim _{n \rightarrow \infty} \max \left\{\frac{\left(3^{s}+1\right) 2^{j s}\|x\|^{s}}{16^{j}}, \frac{\left(2^{s}+1\right) 2^{j s}\|x\|^{s}}{16^{j}}, \frac{2^{s} 2^{j s}\|x\|^{s}}{16^{j}},\right. \\
& \left.\frac{2^{j s} 18\|x\|^{s}}{16^{j}}, \frac{2^{i s} 22\|x\|^{s}}{16^{j}}: 0 \leq i \leq j<n+i\right\} \\
& =\frac{\lambda}{960} \lim _{i \rightarrow \infty} \lim _{n \rightarrow \infty} \frac{2^{i s} 22\|x\|^{s}}{16^{i}}=0
\end{aligned}
$$

and

$$
\begin{aligned}
\lim _{i \rightarrow \infty} \lim _{n \rightarrow \infty} \max \left\{\frac{\psi\left(0,2^{j} x\right)}{2^{j}}: 0 \leq i \leq j<n+i\right\} & =\frac{\lambda}{48} \lim _{i \rightarrow \infty} \lim _{n \rightarrow \infty} \max \left\{\frac{2^{j^{s}}\|x\|^{s}}{2^{j}}: 0 \leq i \leq j<n+i\right\} \\
& =\frac{\lambda\|x\|^{s}}{48} \lim _{i \rightarrow \infty} \lim _{n \rightarrow \infty}\left(2^{s-1}\right)=0 .
\end{aligned}
$$

From (3.88)-(3.91), we can obtain that

$$
\lim _{i \rightarrow \infty} \lim _{n \rightarrow \infty} \max \left\{\frac{\tilde{\psi}\left(2^{j} x\right)}{16^{j}}: 0 \leq i \leq j<n+i\right\}=0
$$

and

$$
\lim _{i \rightarrow \infty} \lim _{n \rightarrow \infty} \max \left\{\frac{\psi\left(0,2^{j} x\right)}{2^{j}}: 0 \leq i \leq j<n+i\right\}=0
$$

for all $x \in G$.

Now, all hypotheses of Theorem 3.3 hold. Therefore, we get this result.

Corollary 3.5. Let $G$ be a normed space and $\left(Y,\|\cdot\|_{Y}\right)$ be a complete non-Archimedean normed space. Suppose that $f: G \rightarrow Y$ is a mapping satisfying $f(0)=0$ and there are a positive real number $\lambda$ and a real number $s<1$ such that

$$
\|D f(x, y)\|_{Y} \leq \lambda\left(\|x\|^{s}\|y\|^{s}+\|x\|^{s}+\|y\|^{s}\right)
$$

for all $x, y \in G-\{0\}$. Then, there exist a unique quartic mapping $Q: G \rightarrow Y$ and a unique additive mapping $A: G \rightarrow Y$ such that

$$
\begin{gathered}
\left\|\frac{f(x)+f(-x)}{2}-Q(x)\right\|_{Y} \leq \frac{\lambda}{960} \lim _{n \rightarrow \infty} \max \left\{\frac{2^{j s}\|x\|^{s}}{16^{j}}\left(3^{s} 2^{j s}\|x\|^{s}+3^{s}+1\right), \frac{2^{j}\|x\|^{s}}{16^{j}}\left(2^{s} 2^{j s}\|x\|^{s}+2^{s}+1\right),\right. \\
\left.\frac{2^{j s+s}\|x\|^{s}}{16^{j} \cdot 2}, \frac{2^{j s} 9\|x\|^{s}}{16^{j}}\left(2^{j s}\|x\|^{s}+2\right), \frac{2^{j s} 22\|x\|^{s}}{16^{j}}: 0 \leq j<n\right\}, \\
\left\|\frac{f(x)-f(-x)}{2}-A(x)\right\|_{Y} \leq \frac{\lambda}{48} \lim _{n \rightarrow \infty} \max \left\{\frac{2^{j s}\|x\|^{s}}{2^{j}}: 0 \leq j<n\right\}
\end{gathered}
$$


and

$$
\begin{aligned}
\|f(x)-Q(x)-A(x)\|_{Y} \leq \max & \left\{\frac { \lambda } { 9 6 0 } \operatorname { l i m } _ { n \rightarrow \infty } \operatorname { m a x } \left\{\frac{2^{j s}\|x\|^{s}}{16^{j}}\left(3^{s} 2^{j s}\|x\|^{s}+3^{s}+1\right),\right.\right. \\
& \frac{2^{j s}\|x\|^{s}}{16^{j}}\left(2^{s} 2^{j s}\|x\|^{s}+2^{s}+1\right), \frac{2^{j s+s}\|x\|^{s}}{16^{j} \cdot 2}, \frac{2^{j s} 9\|x\|^{s}}{16^{j}}\left(2^{j s}\|x\|^{s}+2\right), \\
& \left.\left.\frac{2^{j s} 22\|x\|^{s}}{16^{j}}: 0 \leq j<n\right\}, \frac{\lambda}{48} \lim _{n \rightarrow \infty} \max \left\{\frac{2^{j s}\|x\|^{s}}{2^{j}}: 0 \leq j<n\right\}\right\}
\end{aligned}
$$

for all $x \in G-\{0\}$.

Proof. Define a mapping $\phi: X^{2} \rightarrow[0, \infty)$ by

$$
\phi(x, y)= \begin{cases}0, & \text { if } x=0 \text { or } y=0 \\ \lambda\left(\|x\|^{s}\|y\|^{s}+\|x\|^{s}+\|y\|^{s}\right), & \text { other }\end{cases}
$$

for all $x, y \in G$. By using the same technique with Corollary 3.4, we get this result.

Next, we consider the stability result of (1.5) in non-Archimedean normed spaces by using the fixed point method.

Theorem 3.6. Let $G$ be an additive group, $\left(Y,\|\cdot\|_{Y}\right)$ be a complete non-Archimedean normed space and $\phi: G^{2} \rightarrow Y$ be a function such that

$$
\phi(2 x, 2 y) \leq L \phi(x, y)
$$

for all $x, y \in G$, where $0 \leq L<2$. Suppose that $f: G \rightarrow Y$ is a mapping satisfying $f(0)=0$ and

$$
\|D f(x, y)\|_{Y} \leq \phi(x, y)
$$

for all $x, y \in G$. Then, there exist a unique quartic function $Q: G \rightarrow Y$ and a unique additive function $A: G \rightarrow Y$ such that

$$
\begin{aligned}
& \left\|\frac{f(x)+f(-x)}{2}-Q(x)\right\|_{Y} \leq \frac{1}{960-60 L} \tilde{\psi}(x), \\
& \left\|\frac{f(x)-f(-x)}{2}-A(x)\right\|_{Y} \leq \frac{1}{48-24 L} \tilde{\psi}(x)
\end{aligned}
$$

and

$$
\|f(x)-Q(x)-A(x)\|_{Y} \leq \max \left\{\frac{1}{960-60 L} \tilde{\psi}(x), \frac{1}{48-24 L} \tilde{\psi}(x)\right\}
$$

for all $x \in G$, where $\psi(x, y):=\max \{\phi(x, y), \phi(-x,-y)\}$ for all $x, y \in G$ and

$$
\tilde{\psi}(x):=\max \left\{\psi(3 x, x), \psi(2 x, x), \frac{\psi(0,2 x)}{2}, 9 \psi(x, x), 22 \psi(0, x)\right\}
$$

for all $x, y \in G$.

Proof. Let $\Omega:=\{g: G \rightarrow Y\}$. Define a generalized metric $d$ on $\Omega$ by

$$
d(g, h)=\inf \left\{c \in \mathbb{R}^{+}:\|g(x)-h(x)\| \leq c \tilde{\psi}(x), \forall x \in G\right\} .
$$


Since $Y$ is a complete non-Archimedean normed space, $(\Omega, d)$ is a generalized complete metric space. Let $f_{1}: G \rightarrow Y$ be the function defined by $f_{1}(x):=\frac{f(x)+f(-x)}{2}$ for all $x \in G$. Then, $f_{1}(0)=0$ and $f_{1}(x)=f_{1}(-x)$ for all $x \in G$. By following the proof of Theorem 3.3, we get

$$
\left\|f_{1}(y)-\frac{f_{1}(2 y)}{16}\right\|_{Y} \leq \frac{1}{960} \tilde{\psi}(y)
$$

for all $y \in G$. Define a map $J_{e}: \Omega \rightarrow \Omega$ by $\left(J_{e} g\right)(x)=\frac{f_{1}(2 x)}{16}$ for all $x \in G$. Let $g, h \in \Omega$ and $c$ a positive real number with $d(g, h) \leq c$. Then,

$$
\left\|\left(J_{e} g\right)(x)-\left(J_{e} h\right)(x)\right\|_{Y}=\left\|\frac{g(2 x)}{16}-\frac{h(2 x)}{16}\right\|_{Y}=\frac{1}{16}\|g(2 x)-h(2 x)\|_{Y} \leq \frac{c}{16} \tilde{\psi}(2 x) \leq \frac{c L}{16} \tilde{\psi}(x)
$$

for all $x \in X$ and so

$$
d\left(J_{e} g, J_{e} h\right) \leq \frac{L}{16} d(g, h)
$$

for all $g, h \in \Omega$. Since $\frac{L}{16}<1$, we get $J_{e}$ is strictly contractive and by (3.100), we have

$$
d\left(J_{e} f_{1}, f_{1}\right) \leq \frac{1}{960} .
$$

Hence, by Theorem 2.6, there is the unique fixed point $Q$ of $J_{e}$ in $\Omega$ such that $\left\{J_{e}^{n} f_{1}\right\}$ converges to $Q$ in $(\Omega, d)$ and

$$
d\left(Q, f_{1}\right) \leq\left(\frac{1}{1-L}\right) d\left(J_{e} f_{1}, f_{1}\right) \leq\left(\frac{1}{1-16^{-1} L}\right) \frac{1}{960}=\frac{1}{960-60 L}
$$

for all $x \in X$. By (3.95), we have

$$
\begin{aligned}
\left\|\frac{D f_{1}\left(2^{n} x, 2^{n} y\right)}{16^{n}}\right\|_{Y} & =\frac{1}{2}\left\|\frac{D f\left(2^{n} x, 2^{n} y\right)+D f\left(-2^{n} x,-2^{n} y\right)}{16^{n}}\right\|_{Y} \\
& \leq \frac{1}{2} \max \left\{\frac{\left\|D f\left(2^{n} x, 2^{n} y\right)\right\|_{Y}}{16^{n}}, \frac{\left\|D f\left(-2^{n} x,-2^{n} y\right)\right\|_{Y}}{16^{n}}\right\} \\
& \leq \frac{1}{2} \max \left\{\frac{\phi\left(2^{n} x, 2^{n} y\right)}{16^{n}}, \frac{\phi\left(-2^{n} x,-2^{n} y\right)}{16^{n}}\right\} \\
& \leq \frac{L^{n}}{2 \cdot 16^{n}} \max \{\phi(x, y), \phi(-x,-y)\}
\end{aligned}
$$

for all $x, y \in G$. From the last inequality, letting $n \rightarrow \infty$, we obtain

$$
D Q(x, y)=0
$$

for all $x, y \in G$. Since $f_{1}(0)=0$, we obtain $Q(0)=0$. Since $f_{1}$ is even, we obtain

$$
\|Q(x)-Q(-x)\|_{Y}=\lim _{n \rightarrow \infty}\left\|Q(x)-J_{e}^{n} f_{1}(-x)\right\|_{Y}=\lim _{n \rightarrow \infty}\left\|Q(x)-J_{e}^{n} f_{1}(x)\right\|_{Y}=0
$$

for all $x \in G$ and so $Q$ is even. By Lemma 3.2, we have $Q$ is a quartic mapping. From (3.101), we get

$$
\left\|\frac{f(x)+f(-x)}{2}-Q(x)\right\|_{Y} \leq \frac{1}{960-60 L} \tilde{\psi}(x)
$$

for all $x \in G$.

Let $f_{2}: G \rightarrow Y$ be the function defined by $f_{2}(x):=\frac{f(x)-f(-x)}{2}$ for all $x \in G$. Then, $f_{2}(0)=0$ and $f_{2}(-x)=-f_{2}(x)$ for all $x \in G$. From the proof of Theorem 3.3, we get

$$
\left\|f_{2}(y)-\frac{f_{2}(2 y)}{2}\right\|_{Y} \leq \frac{\psi(0, y)}{48} \leq \frac{\tilde{\psi}(y)}{48}
$$


for all $y \in G$. Define a map $J_{o}: \Omega \rightarrow \Omega$ by $\left(J_{o} g\right)(x)=2 g\left(2^{-1} x\right)$ for all $x \in X$. Let $g, h \in \Omega$ and $c$ a positive real number with $d(g, h) \leq c$. Then,

$$
\left\|\left(J_{o} g\right)(x)-\left(J_{o} h\right)(x)\right\|_{Y}=\left\|\frac{g(2 x)}{2}-\frac{h(2 x)}{2}\right\|_{Y}=\frac{1}{|2|}\|g(2 x)-h(2 x)\|_{Y} \leq \frac{c}{|2|} \tilde{\psi}(2 x) \leq \frac{c L}{|2|} \tilde{\psi}(x)
$$

for all $x \in G$ and so

$$
d\left(J_{o} g, J_{o} h\right) \leq \frac{L}{2} d(g, h)
$$

for all $g, h \in \Omega$. Since $2^{-1} L<1$, we get $J_{e}$ is strictly contractive and by (3.104), we have

$$
d\left(J_{o} f_{2}, f_{2}\right) \leq \frac{1}{48} .
$$

Hence, by Theorem 2.6, there is the unique fixed point $A$ of $J_{o}$ in $\Omega$ such that $\left\{J_{o}^{n} f_{2}\right\}$ converges to $A$ in $(\Omega, d)$ and

$$
d\left(A, f_{2}\right) \leq\left(\frac{1}{1-2^{-1} L}\right) d\left(J_{o} f_{2}, f_{2}\right) \leq\left(\frac{1}{1-2^{-1} L}\right) \frac{1}{48}=\frac{1}{48-24 L}
$$

for all $x \in G$. By (3.95), we have

$$
\begin{aligned}
\left\|2^{-n} D f_{2}\left(2^{n} x, 2^{n} y\right)\right\|_{Y} & =\frac{1}{2}\left\|\frac{D f\left(2^{n} x, 2^{n} y\right)+D f\left(-2^{n} x,-2^{n} y\right)}{2^{n}}\right\|_{Y} \\
& \leq \frac{1}{2} \max \left\{\frac{\left\|D f\left(2^{n} x, 2^{n} y\right)\right\|_{Y}}{2^{n}}, \frac{\left\|D f\left(-2^{n} x,-2^{n} y\right)\right\|_{Y}}{2^{n}}\right\} \\
& \leq \frac{1}{2} \max \left\{\frac{\phi\left(2^{n} x, 2^{n} y\right)}{2^{n}}, \frac{\phi\left(-2^{n} x,-2^{n} y\right)}{2^{n}}\right\} \\
& \leq \frac{L^{n}}{2^{n+1}} \max \{\phi(x, y)+\phi(-x,-y)\}
\end{aligned}
$$

for all $x, y \in G$. Letting $n \rightarrow \infty$ in the last inequality, we have

$$
D A(x, y)=0
$$

for all $x, y \in X$. Since $f_{2}(0)=0$, so we obtain $A(0)=0$. Since $f_{2}$ is odd,

$$
\|A(x)+A(-x)\|_{Y}=\lim _{n \rightarrow \infty}\left\|A(x)+J_{o}^{n} f_{2}(-x)\right\|_{Y}=\lim _{n \rightarrow \infty}\left\|A(x)-J_{o}^{n} f_{2}(x)\right\|_{Y}=0
$$

for all $x \in G$ and so $A$ is odd. By Lemma 3.2, we have $A$ is an additive mapping. From (3.105), we get

$$
\left\|\frac{f(x)-f(-x)}{2}-A(x)\right\|_{Y} \leq \frac{1}{48-24 L} \tilde{\psi}(x)
$$

for all $x \in G$. Since $f(x)=f_{1}(x)+f_{2}(x)$ for all $x \in G$, from (3.103) and (3.107) it follows that

$$
\begin{aligned}
\|f(x)-Q(x)-A(x)\| & =\left\|f_{1}(x)+f_{2}(x)-Q(x)-A(x)\right\|_{Y} \\
& \leq \max \left\{\left\|f_{1}(x)-Q(x)\right\|_{Y},\left\|f_{2}(x)-A(x)\right\|_{Y}\right\} \\
& \leq \max \left\{\frac{1}{960-60 L} \tilde{\psi}(x), \frac{1}{48-24 L} \tilde{\psi}(x)\right\}
\end{aligned}
$$

for all $x \in G$.

To show the uniqueness of $Q$ and $A$, suppose that there exist a quartic mapping $Q^{\prime}: G \rightarrow Y$ satisfying (3.97) and an additive $A^{\prime}: G \rightarrow Y$ satisfying (3.98). By using (3.97), we have 


$$
\begin{aligned}
\left\|Q(x)-Q^{\prime}(x)\right\|_{Y} & =\lim _{n \rightarrow \infty}\left\|\frac{Q\left(2^{n} x\right)}{2^{n}}-\frac{Q^{\prime}\left(2^{n} x\right)}{16^{n}}\right\|_{Y} \\
& =\lim _{n \rightarrow \infty} \frac{1}{16^{i}}\left\|Q\left(2^{n} x\right)-f_{1}\left(2^{n} x\right)-Q^{\prime}\left(2^{n} x\right)+f_{1}\left(2^{n} x\right)\right\|_{Y} \\
& \leq \lim _{n \rightarrow \infty} \frac{1}{16^{i}} \max \left\{\left\|Q\left(2^{n} x\right)-f_{1}\left(2^{n} x\right)\right\|_{Y},\left\|Q^{\prime}\left(2^{n} x\right)-f_{1}\left(2^{n} x\right)\right\|_{Y}\right\} \\
& \leq \frac{1}{960-60 L} \lim _{n \rightarrow \infty} \frac{1}{16^{n}} \max \left\{\tilde{\psi}\left(2^{n} x\right)\right\} \\
& \leq \frac{1}{960-60 L} \lim _{n \rightarrow \infty} \frac{L^{n}}{16^{n}} \tilde{\psi}(x)
\end{aligned}
$$

for all $x \in G$. Since the right-hand side of the above inequality converges to 0 , we obtain $Q(x)=Q^{\prime}(x)$ for all $x \in G$, that is, $Q=Q^{\prime}$. Similarly, we get $A=A^{\prime}$.

Corollary 3.7. Let $G$ be a normed space and $\left(Y,\|\cdot\|_{Y}\right)$ be a complete non-Archimedean normed space. Suppose that $f: G \rightarrow Y$ is a mapping satisfying $f(0)=0$, and there are a positive real number $\lambda$ and a real number $s<1$ such that

$$
\|D f(x, y)\|_{Y} \leq \lambda\left(\|x\|^{S}+\|y\|^{S}\right)
$$

for all $x, y \in G-\{0\}$. Then, there exist a unique quartic function $Q: G \rightarrow Y$ and a unique additive mapping $A: G \rightarrow Y$ such that

$$
\begin{aligned}
& \left\|\frac{f(x)+f(-x)}{2}-Q(x)\right\|_{Y} \leq \frac{22 \lambda\|x\|^{s}}{960-2^{s} 60}, \\
& \left\|\frac{f(x)-f(-x)}{2}-A(x)\right\|_{Y} \leq \frac{22 \lambda\|x\|^{s}}{48-2^{s} 24}
\end{aligned}
$$

and

$$
\|f(x)-Q(x)-A(x)\|_{Y} \leq \max \left\{\frac{22 \lambda\|x\|^{s}}{960-2^{s} 60}, \frac{22 \lambda\|x\|^{s}}{48-2^{s} 24}\right\}
$$

for all $x \in G-\{0\}$.

Proof. Define a mapping $\phi: G^{2} \rightarrow[0, \infty)$ by

$$
\phi(x, y)= \begin{cases}0, & \text { if } x=0 \text { or } y=0 ; \\ \lambda\left(\|x\|^{s}+\|y\|^{S}\right), & \text { other }\end{cases}
$$

for all $x, y \in G$.

Next, we will show that $\phi(2 x, 2 y) \leq L \phi(x, y)$ for all $x, y \in G$, where $L:=2^{s}<2$. For $x=0$ or $y=0$, we get

$$
\phi(2 x, 2 y)=0 \leq L \phi(x, y) .
$$

If $x, y \in G-\{0\}$, we obtain

$$
\phi(2 x, 2 y)=\lambda\left(\|2 x\|^{S}+\|2 y\|^{S}\right)=\lambda 2^{s}\left(\|x\|^{S}+\|y\|^{S}\right)=L \phi(x, y) .
$$

So $\phi(2 x, 2 y) \leq L \phi(x, y)$ for all $x, y \in G$.

Therefore, all hypotheses of Theorem 3.6 hold and so we get this result.

Corollary 3.8. Let $G$ be a normed space and $\left(Y,\|\cdot\|_{Y}\right)$ be a complete non-Archimedean normed space. Suppose that $f: G \rightarrow Y$ is a mapping satisfying $f(0)=0$ and there are a positive real number $\lambda$ and a real number $s<1$ such that

$$
\|D f(x, y)\|_{Y} \leq \lambda\left(\|x\|^{S}\|y\|^{S}+\|x\|^{S}+\|y\|^{S}\right)
$$


for all $x, y \in G-\{0\}$. Then, there exist a unique quartic function $Q: G \rightarrow Y$ and a unique additive mapping $A: G \rightarrow Y$ such that

$$
\begin{aligned}
& \left\|\frac{f(x)+f(-x)}{2}-Q(x)\right\|_{Y} \leq \frac{\lambda\|x\|^{s}}{960-2^{s} 60} \max \left\{3^{s}\|x\|^{s}+3^{s}+1,2^{s}\|x\|^{s}+2^{s}+1,9\|x\|^{s}+18,22\right\}, \\
& \left\|\frac{f(x)-f(-x)}{2}-A(x)\right\|_{Y} \leq \frac{\lambda\|x\|^{s}}{48-2^{s} 24} \max \left\{3^{s}\|x\|^{s}+3^{s}+1,2^{s}\|x\|^{s}+2^{s}+1,9\|x\|^{s}+18,22\right\}
\end{aligned}
$$

and

$$
\begin{aligned}
\|f(x)-Q(x)-A(x)\|_{Y} \leq \max \{ & \frac{\lambda\|x\|^{s}}{960-2^{s} 60} \max \left\{3^{s}\|x\|^{s}+3^{s}+1,2^{s}\|x\|^{s}+2^{s}+1,9\|x\|^{s}+18,22\right\}, \\
& \left.\frac{\lambda\|x\|^{s}}{48-2^{s} 24} \max \left\{3^{s}\|x\|^{s}+3^{s}+1,2^{s}\|x\|^{s}+2^{s}+1,9\|x\|^{s}+18,22\right\}\right\}
\end{aligned}
$$

for all $x \in G-\{0\}$.

Proof. Define a mapping $\phi: G^{2} \rightarrow[0, \infty)$ by

$$
\phi(x, y)= \begin{cases}0, & \text { if } x=y=0 \\ \lambda\left(\|x\|^{s}\|y\|^{s}+\|x\|^{s}+\|y\|^{s}\right), & \text { other }\end{cases}
$$

for all $x, y \in G$. Next, we will show that $\phi(2 x, 2 y) \leq L \phi(x, y)$ for all $x, y \in G$, where $L:=2^{s}<2$. For $x=0$ or $y=0$, we get

$$
\phi(2 x, 2 y)=0 \leq L \phi(x, y) .
$$

If $x, y \in G-\{0\}$, we obtain

$$
\begin{aligned}
\phi(2 x, 2 y) & =\lambda\left(\|2 x\|^{S}\|2 y\|^{S}+\|2 x\|^{S}+\|2 y\|^{S}\right)=\lambda\left(2^{2 s}\|x\|^{S}\|y\|^{s}+2^{s}\|x\|^{S}+2^{s}\|y\|^{s}\right) \\
& \leq \lambda 2^{s}\left(\|x\|^{s}\|y\|^{S}+\|x\|^{s}+\|y\|^{s}\right)=L \phi(x, y) .
\end{aligned}
$$

So $\phi(2 x, 2 y) \leq L \phi(x, y)$ for all $x, y \in G$.

Therefore, all hypotheses of Theorem 3.6 hold and so we get this result.

\section{Conclusion and recommendation}

Two main generalized Hyers-Ulam stability results for the additive-quartic functional equation in the case of an unknown function map from an additive group into a complete non-Archimedean normed space are given based on the direct method and the fixed point method. Several stability results can be obtained from two main results by choosing an appropriate control function $\phi$. In this article, the main second result can be deduced from the fixed point theorem of Diaz and Margolis [18]. However, in 2012, Ciepliński [19] presented that how to use different fixed point theorems with Diaz and Margolis for proving HyersUlam stability of functional equations. The obtained stability results are simple consequences of some new fixed point theorems. Consequently, we give the following challenge as the recommendation to the reader:

Can we use the fixed point results of Cieplinski [19] to prove the stability results for the additive-quartic functional equation whenever an unknown function maps from an additive group into a complete nonArchimedean normed space? 
Acknowledgments: Wutiphol Sintunavarat would like to thank the Thailand Research Fund and Office of the Higher Education Commission under grant no. MRG6180283 for financial support during the preparation of this manuscript. Also, this article was supported by the Thammasat University Research Fund, Contract No. TUGG 36/2562.

\section{References}

[1] S. M. Ulam, Problems in Modern Mathematics, Wiley, New York, 1964.

[2] D. H. Hyers, On the stability of the linear functional equation, Proc. Natl. Acad. Sci. U. S. A. 27 (1941), no. 4, 222-224, DOI: $10.1073 /$ pnas.27.4.222.

[3] Tosio Aoki, On the stability of the linear transformation in Banach spaces, J. Math. Soc. Jpn. 2 (1905), no. 1-2, 64-66, DOI: $10.2969 / \mathrm{jmsj} / 00210064$.

[4] Themistocles M. Rassias, On the stability of the linear mapping in Banach spaces, Proc. Am. Math. Soc. 72 (1978), no. 2, 297-300, DOI: 10.2307/2042795.

[5] John Michael Rassias, Solution of the Ulam stability problem for quartic mappings, Glas. Mat. Ser. 34 (1999), no. 2 , 243-252.

[6] Jukang K. Chung and Prasanna K. Sahoo, On the general solution of a quartic functional equation, Bull. Korean Math. Soc. 40 (2003), no. 4, 565-576, DOI: 10.4134/BKMS.2003.40.4.565.

[7] Prasanna K. Sahoo, On a functional equation characterizing polynomials of degree three, Bull. Inst. Math. Acad. Sin. 32 (2004), no. 1, 35-44.

[8] Montakarn Petapirak and Paisan Nakmahachalasint, $A$ quartic functional equation and its generalized Hyers-UlamRassias stability, Thai J. Math. 6 (2008), no. 3, 77-84.

[9] M. Eshaghi Gordji, Stability of a functional equation deriving from quartic and additive functions, Bull. Korean Math. Soc. 47 (2010), no. 3, 491-502, DOI: 10.4134/BKMS.2010.47.3.491.

[10] Charinthip Hengkrawit and Anurak Thanyacharoen, $A$ general solution of a generalized quartic functional equation and its stability, Int. J. Pure Appl. Math. 80 (2013), no. 4, 691-706, DOI: 10.12732/ijpam.v85i4.6.

[11] Jeongwook Chang, Chang-Kwon Choi, Jooyoung Kim, and Prasanna K. Sahoo, Stability of the cosine-sine functional equation with involution, Adv. Oper. Theory 2 (2017), no. 4, 531-546, DOI: 10.22034/aot.1706-1190.

[12] Chang-Kwon Choi, Jaeyoung Chung, Riedel Thomas, and Prasanna K. Sahoo, Stability of functional equations arising from number theory and determinant of matrices, Ann. Funct. Anal. 8 (2017), no. 3, 329-340, DOI: 10.1215/20088752$0000017 \mathrm{X}$

[13] Charinthip Hengkrawit and Anurak Thanyacharoen, $A$ generalized additive-quartic functional equation and its stability, Bull. Korean Math. Soc. 52 (2015), no. 6, 1759-1776, DOI: 10.4134/BKMS.2015.52.6.1759.

[14] Von K. Hensel, Uber eine neue Begrundung der Theorie der algebraischen Zahlen, Jahres-ber. Deutsch. Math. 6 (1897), 83-88.

[15] L. M. Arriola and W. A. Beyer, Stability of the Cauchy functional equation over p-adic fields, Real Anal. Exchange 31 (2005/ 2006), no. 1, 125-132.

[16] Z. Kaiser, On stability of the Cauchy equation in normed spaces over fields with valuation, Publ. Math. Debrecen 64 (2004), no. 1-2, 189-200.

[17] Mohammad Sal Moslehian and Themistocles M. Rassias, Stability of functional equations in non-Archimedean spaces, Appl. Anal. Discrete Math. 1 (2007), no. 2, 325-334, DOI: 10.2298/AADM0702325M.

[18] J. B. Diaz and Beatriz Margolis, A fixed point theorem of the alternative for contractions on a generalized complete metric space, Bull. Am. Math. Soc. 74 (1968), no. 2, 305-309.

[19] Krzysztof Cieplinski, Applications of fixed point theorems to the Hyers-Ulam stability of functional equations - a survey, Ann. Funct. Anal. 3 (2012), no. 1, 151-164, DOI: 10.15352/afa/1399900032. 